\title{
The Relationship of Musical and Color Preferences of a Person in Computer Graphics
}

\author{
Anastasia Tonkoglaz ${ }^{1}$, Evgeniya Vekhter ${ }^{1}$ and Aleksey Shklyar ${ }^{1}$ \\ ${ }^{1}$ Tomsk Polytechnic University, 30, Lenin Ave., Tomsk, 634050, Russian Federation
}

\begin{abstract}
User-oriented design allows to develop design solutions based on user's needs and desires. In order to meet user's needs better, designers work on understanding and adaptation of human perception principles. Later this data would be used in design processes. The article is relevant due to the problem of interrelation of perceived information about environmental objects through different channels. The existence of this relation is confirmed by empirical studies, but its features and nature are not fully clarified. This article is devoted to the search and analysis of the relationship of information, which was received from different perception channels. This information allows to get input data about client's color and musical preferences. The article considers the comparability of colors and sound, which were obtained by testing a group of respondents. The work includes selection of associative color series for a fragment of a musical composition, which allows to analyze results and to make conclusions about its coincidence and discrepancy. Obtained results show connection of music and color based on artistic and figurative basis. Coincidences can be used in computer graphics while color spaces composing and in design at the stage of coloristic selection.
\end{abstract}

\section{Keywords}

Color schemes, color perception, sound perception, synesthesia, design, coloristics

\section{Theoretical aspects of color and music preferences}

Sensory design is focused on the formation and analysis of general spectrum of perception by senses. Through sensory analysis, a designer can improve a designed object and affect user experience. Sensory design includes the analysis of interaction with objects and the environment. The main purpose of the observation is to determine user opinion about a human product, its positive and negative aspects in terms of tactility, appearance, acoustic qualities. The founder of the developing field of sensory research is David Howes - professor of anthropology and director of the Center of Sensory Research at Concordia University) [1].

Using different sensory dimensions, designers achieve a variety of user interactions and experiences. For example, maps that can be touched and viewed form an accessible object for sighted people and also for visually impaired and blind users. Audio devices convert sound into vibrations that could be felt by skin. Dishes and kitchen utensils have special color and shape to guide people suffering from dementia or vision loss. These tools are useful for all users, because sensory design increases user's awareness of the environment and forms a diverse user experience, stimulating internal reactions.

Understanding sound as a sensory experience in design can change a person's perception of objects, how he analyzes his experience and what he feels. This can help to empower a designer to create a holistic user experience. Human sensory organs are channels that provide him a contact with the external environment. These channels are inextricably linked. Each of them differs from the other in the amount of information that passes per unit of time. The more developed the sense organ is, the more it distinguishes effects of the external environment, the more information passes through it.

GraphiCon 2021: 31st International Conference on Computer Graphics and Vision, September 27-30, 2021, Nizhny Novgorod, Russia EMAIL: as.tonkoglaz@gmail.com (A. Tonkoglaz); vehter@tpu.ru (E. Vekhter); shklyarav@tpu.ru (A. Shklyar) ORCID: 0000-0002-0246-1068 (A. Tonkoglaz); 0000-0003-0604-0399 (E. Vekhter); 0000-0003-4442-7420 (A. Shklyar) (c) (i) 2021 Copyright for this paper by its authors.

Use permitted under Creative Commons License Attribution 4.0 International (CC BY 4.0).

CEUR Workshop Proceedings (CEUR-WS.org) 
Since the time of I. Newton, attempts have been made to establish the visual equivalent of sound on the principle of universality of color-sound correspondences. Numerous experiments of color and music synthesis indicate a steady interest in this phenomenon. Philosophers, mathematicians, physicists, inventors and artists have tried to explain this phenomenon. Of course, this indicates the significance of the idea of connecting and interacting visual and auditory sensations. It reveals multifaceted approaches to the search of music and color synthesis, despite of the diversity of light and music experiments.

Fred Collopy is a professor of design and innovation at Case Western Reserve University. His works are published in the field of designing visual design tools, design process management, information technology methodology [2]. In 1998, emerat professor Fred Collopy founded the Rhythmic Light project as a place of results concentration of "visual music" research [3]. In his project, Fred Collopy studies connections between acoustic and visual means, systematizes existing knowledge and hypotheses. Looking back on previous experience, it is important to analyze the general trend of analyzing sound and color synthesis.

Within this work, Fred Collopy's diagram is taken for analysis, in which color and musical analogies of different representatives of color music of XVIII-XXI centuries are systematized (Figure 1) [3]. Main representatives of color music are organized in a time sequence. It describes their hypotheses about the ratio of color and sound series. Color combinations to the note row are shown on the right. Within this article some representatives of this table from various fields of science and art were considered.

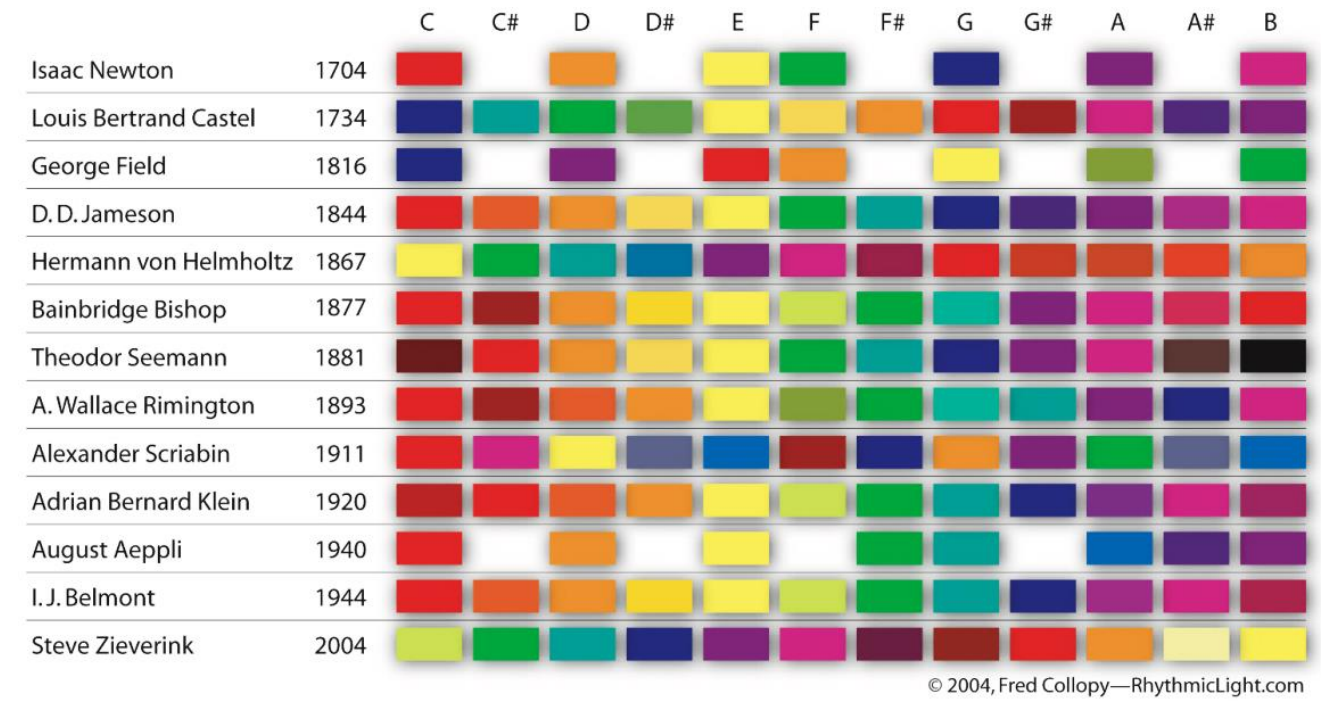

Figure 1: Color and musical analogies

The scientific basis of light music was laid by Isaac Newton [4], who discovered an analogy between the solar spectrum, divided into seven parts (the law of refraction of light), and the musical scale. As a result of this ratio, each musical sound of the scale corresponded to a certain color of the spectrum. The scientist discovered this system by comparing lengths of colored sections of the spectrum with ratios of vibrations frequencies of musical tones. The division of the spectrum into seven color zones is conditional.

Louis Bertrand Castel included his own scheme, which was different from the previous one, in his color organ [5]. Castel proposed a color-music technology that uses diffuse reflection of light from a pigment.

Chemist George Field built a color circle based on basic colors of red, yellow and blue, thus he wanted to take the opposite position to Newton [6]. Secondary and tertiary colors arise through subtractive overlay.

Hermann Helmholtz's theory is a theory of color perception, which assumes the existence of special elements in the eye for perception of red, green and blue colors [7]. Spectral colors shine more intensely and have greater saturation. They are mixed additively, while the pigments are mixed subtractively. Helmholtz presents 3 variables that are still used to characterize color: tone, saturation and brightness. These characteristics of color sensations were chosen in order to correspond to three parameters of sound: strength, height and timbre. The only difference between a sound phenomenon and color 
perception is that the eye cannot distinguish components of a mixed color, while the ear allows to separate elements of a complex sound.

The Russian composer A. N. Scriabin is considered as a bright representative of light and music art. The composer had a color ear, which gives a sense of color during the performance of music. Alexander Scriabin compared color and sound based on his synesthetic sensations, without using physical indicators of both categories [8]. This is reflected in his color range, which is clearly different from representatives of scientific fields. He decided to embody his visual perception in the poem "Prometheus" or " The Poem of Fire "in 1910, adding a line of Luce light to the score, which he planned to implement through" tastiera per luce "("light keyboard"). The involvement of many organs of human perception was not the goal, but a mean of figurative influence on a viewer.

Analyzing visual and sound correspondences, we can conclude that light music can act as a multifactorial mean of perception. Factors, which influence color-sound combinations are variable: depending on the scientific or artistic-figurative justification, on the volume of accumulated scientific ideas of each period, on the level of development of technical capabilities, which are necessary for experimental experience and implementation in art. Light music contributes to the development of imaginative and associative thinking and can be used as a tool for influencing the viewer, which allows to combine and compare incoming information about an object or an environment.

\section{Practical part: conducting comparisons}

Color, along with the objective effect on the organ of vision, causes a subjective reaction of a person based on their own color preferences. To find out the nature of color preferences and associative images, it is necessary to compare the subjective reaction to the same sound stimulus (a musical fragment).

To achieve this the testing was carried out. The testing was attended by 20 people aged 17 to 25 years, who were divided into 2 groups according to their fields of activity: the field of design and fine arts (Group 10), and musical creativity (Group 2). The survey participants were presented with 25 music fragments with the same duration of 15 seconds. Participants indicated the degree of sympathy for each of them on a nine-point scale from "disgusting" to "amazing". After determination high-valuable fragments of each respondent, participants had to choose a color to the selected fragment at the next listening. Used music samples belonged to different musical genres and were unfamiliar to respondents.

The survey participants of the first group selected compositions related to the directions: alternative rock (3 people), symphonic music ( 1 person), pop music (4 persons), electronic music ( 2 persons). The survey participants of the second group selected compositions related to the directions: electronic music (4 persons), alternative rock ( 2 persons), pop music ( 3 persons), country ( 1 person).

After analysis of the selected colors, the conclusion is, that the main difference between two groups is, that respondents of the second group chose colors close to the main ones (35\% are main colors). By mixing this colors, it is possible to get all other colors and shades (Figure 2). Participants from the first group mostly preferred complex colors: shades that are obtained from the main colors of the spectrum when black is added to them or when more than two main colors are mixed ( $90 \%$ are complex colors). In the first group, the main choice is formed in the direction of warm, light colors (60\%), in the second group, cold and saturated colors are more common (57.5\%). The main color harmonies are: analogy a group of three colors that are next to each other on the color wheel, and monochrome color schemes. 


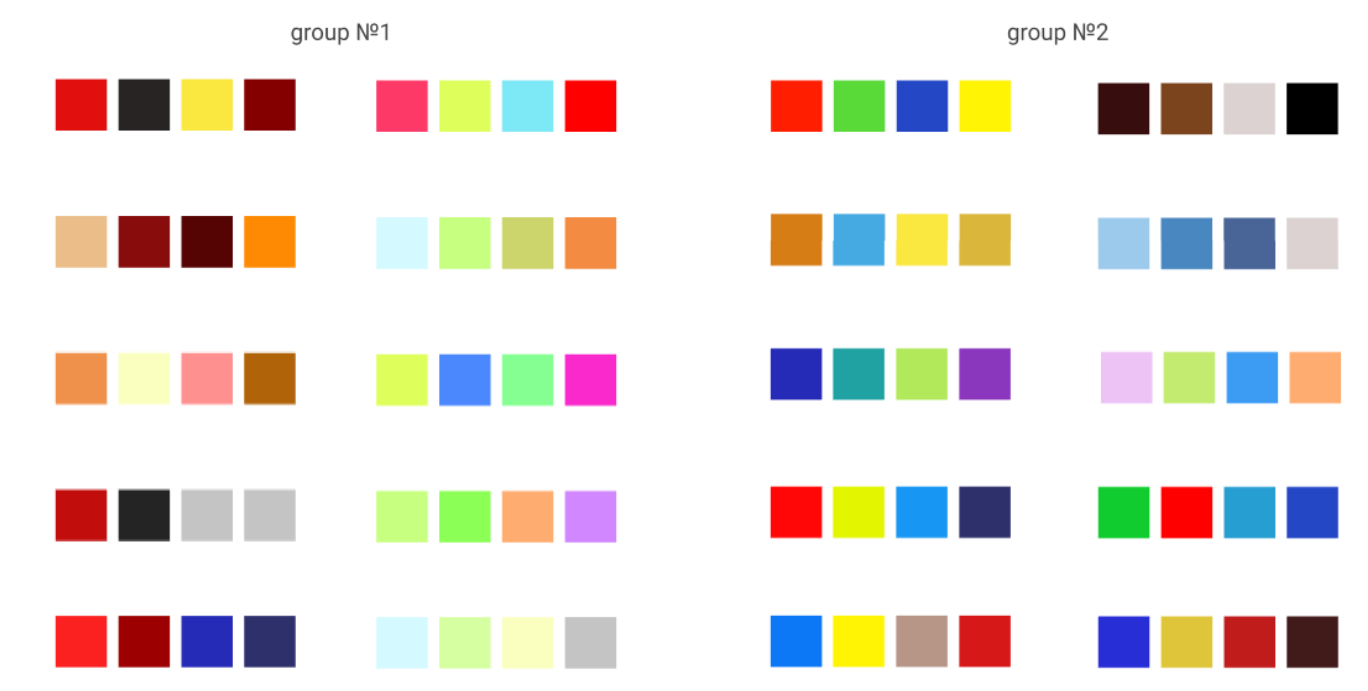

Figure 2: Color and musical analogies

While analyzing results of the survey, color comparisons of respondents to the same musical fragment belonging to the "alternative rock" genre were considered. In the survey, 2 participants from each group rated this sample equally high, which allows to compare their results, to find similarities and differences (Figure 3).

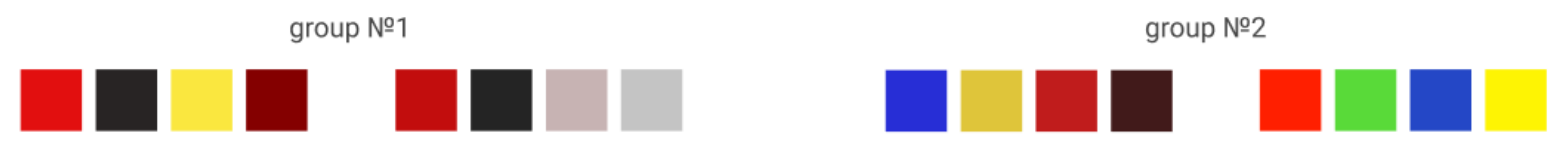

Figure 3: Color and musical analogies to the same fragment

Participants from the first group preferred dark, red colors and there are analogies to them, the respondents of the second group explained the chosen colors by the "brightness" of the musical composition. Red is found in all variants, in 3 out of 4 variants red colors are located in the first place, and 3 out of 4 variants contain yellow shades.

This result indicates that regardless of the major of the group members, an equally highly rated musical composition can cause similar color associations, regardless of their subjective associations.

\section{Conclusion}

In the process of selecting the color content, respondents of both groups operated by internal associations and images formed during listening to the selected musical fragment. Obtained color-sound correspondences are, with the exception of individual cases, variable in nature, but are determined by the stable individual ideas.

People put different, and sometimes contradictory values in the same colors. Through tests and subsequent analysis, it is possible to develop ways of obtaining information about a person, about his subjective perception of color [9]. The results obtained at this stage indicate that through the relationship of sound and color, it is possible to obtain information about the client's wishes for color and musical preferences.

Scientific research in the field of the connection of sound and color, its influence has been carried out repeatedly, as an example, there are works of physicist and physiologist academician P. P. Lazarev, psycholinguist A. P. Zhuravlev. However, they did not get wide practical use in design field. Now, using computer graphics tools, it is possible to determine accurately stable individual color preferences and obtain complex characteristics based on a person's musical sympathies. Color schemes, which were identified with the help of computer graphics tools, have a number of advantages in color purity and in color range. 
Obtained information might be used in design at the stage of coloristic selection. For work on an individual order, it is difficult to get a final color solution based only on analogues presented by the customer. In this case, it is necessary to analyze the nature of several color comparisons to different musical fragments, abstracting from references, concentrating on internal images: are there color harmonies in them, if so, which ones? Does he consciously or unconsciously operate with them? How many colors are used to convey color associations? Basing on this, it is necessary to determine frequently used colors based on different musical samples, which colors are not present at all, a tendency to warm or cold tones and other features. Which contrasts and accents are found. The analysis of tracking matches will allow the designer to get information about the customer's color preferences and suggest the most attractive option for him.

\section{Acknowledgements}

The reported study was funded by RFBR, project number 19-07-00844.

\section{References}

[1] D. Howes, Sensory Studies, 2006. URL: http://www.sensorystudies.org.

[2] F. Collopy, Color, Form, and Motion: Dimensions of a Musical Art of Light, Leonardo (2000) 33 (5): $355-360$

[3] F. Collopy, Rhythmic light, hue to pitch, 2004. URL: https://rhythmiclight.com/visual-musiccorrespondences/hue-to-pitch/.

[4] I. Newton, Opticks or, a Treatise of the Reflections, Refractions, Inflections, and Colours of Light, William\&John Innys, 1721

[5] M. Franssen, "The Ocular Harpsichord of Louis-Bertrand Castel," Sci. Aesthet. An EighteenthCentury Cause Célèbre, pp. 15-77, 1991.

[6] G. Field, Field's Chromatography or Treatise on Colours and Pigments as Used by Artists, London : Windsor and Newton, 1885

[7] H. von Helmholtz, Treatise on Physiological Optics, Courier Corporation, Volume II, 2013.

[8] I. Belza, Alexander Nikolaevich Scriabin, Moscow, Music, 1983.

[9] A.A. Zakharova, E.V. Vekhter, A.V. Shklyar, Adaptable Visualization. Scientific Visualization 13 (4), 67-78 (2021). doi: 10.26583/sv.13.2.05 